\title{
On Energy-Efficient Time Synchronization for Wireless Sensors under Large-Scale and Small-Scale Fading Effects
}

\author{
Pablo Briff ${ }^{1}$, Ariel Lutenberg ${ }^{1,2}$, Leonardo Rey Vega ${ }^{2,3}$, Fabian Vargas ${ }^{4}$ \\ ${ }^{1}$ Embedded Systems Laboratory, Faculty of Engineering, University of Buenos Aires, Buenos Aires, Argentina \\ ${ }^{2}$ CONICET, National Scientific and Technical Research Council, Buenos Aires, Argentina \\ ${ }^{3}$ LPSC, Faculty of Engineering, University of Buenos Aires, Buenos Aires, Argentina \\ ${ }^{4}$ Signals and Systems for Computing Group, Faculty of Engineering, Catholic University, Porto Alegre, Brazil \\ Email: pbriff@fi.uba.ar, lse@fi.uba.ar, lrey@fi.uba.ar, vargas@pucrs.br
}

Received July 27, 2013; revised August 27, 2013; accepted September 4, 2013

Copyright (C) 2013 Pablo Briff et al. This is an open access article distributed under the Creative Commons Attribution License, which permits unrestricted use, distribution, and reproduction in any medium, provided the original work is properly cited.

\begin{abstract}
In this work, the existing trade-off between time synchronization quality and energy is studied for both large-scale and small-scale fading wireless channels. We analyze the clock offset estimation problem using one-way, two-way and $\mathrm{N}$-way message exchange mechanisms affected by Gaussian and exponentially distributed impairments. Our main contribution is a general relationship between the total energy required for synchronizing a wireless sensor network and the clock offset estimation error by means of the transmit power, number of transmitted messages and average message delay, deriving the energy optimal lower bound as a function of the time synchronization quality and the number of hops in a multi-hop network.
\end{abstract}

Keywords: Wireless Sensor Networks; Clock Offset Estimation; Time Synchronization; Wireless Channel Fading

\section{Introduction}

With the advent of wireless technologies over the last decade, Wireless Sensor Networks (WSN's) are overtaking wired networks in the field of sensing [1]. A WSN typically consists of low cost sensor nodes that run on battery or obtain energy from harvesting techniques, namely self-powered sensors. For this reason, energy management becomes a substantial matter in order to guarantee reasonable sensors' lifetime values. Networkwide time synchronization for WSN's provides mechanisms for achieving power management and device location, while it constitutes an indispensable requirement for in-network processing of the sensed data. However, synchronizing an entire WSN represents a far-from-trivial enterprise that encompasses a suite of challenges to be solved, for which it remains within the top-five most challenging open topics in WSN's [2]. Still, with the recent introduction of the "Internet of Things" (IoT), the density of the nodes in a WSN is expected to be considerably increased [3], and time synchronization will become a substantial need for achieving the desired performance figures. Due to the fact that in the most general case there will be no common clock signal broadcasted over the entire network, time synchroniza- tion algorithms aim to provide a mechanism by which all sensors obtain an estimate of their internal clocks with respect to the other nodes so as to reach a consensus on the concept of time among all the sensors in the network. For each sensor $i$, its internal clock $c_{i}$ can be modelled as a linear equation with a corresponding skew $\alpha_{i}$ and offset $\beta_{i}$ [4,5], namely $c_{i}(t)=\alpha_{i} \cdot t+\beta_{i}$. In order to achieve a target synchronization quality, parameter estimation techniques can be applied, being the estimation error $\varepsilon$ a function of the estimator and the number of samples employed. Since time synchronization involves messages exchange and transmission/reception operation, it becomes an energy-consuming task for sensors to carry out; still, the communication channel may undergo impairments making transmitted messages do not reach their destinations, which constitutes a waste of energy for the entire network. Consequently, time synchronization implies an unavoidable energy expenditure; however, when achieved, it could allow significant further savings in energy consumption through proper network power management.

This work is organized as follows: Section 2 provides an overview of the related work on the field of clock offset estimation, Sections 3 and 4 state the general system model for the power consumption vs. estimation 
quality trade-off when using one-way messages, Section 5 extends the limits found in Section 4 to two-way messages' clock offset estimation, and a generalization of the problem to n-way messages is presented in Section 6; Section 7 shows that the results for two-way messages exchange are applicable to the renowned ReferenceBroadcast Synchronization algorithm [6], whereas Section 8 depicts the simulation results for the limits found in this research for the two-way message exchange scenario. Finally, Section 9 exposes the conclusions of this work.

\section{Related Work}

There is a number of clock synchronization techniques that base their operating principle on estimation theory and messages exchange among sensors. Examples of these are Reference-Broadcast Synchronization (RBS) [6], Timing-Sync Protocol for Sensor Networks (TPSN) [7] and Pairwise Broadcast Synchronization (PBS) [8]. All three protocols make use of the number of received messages from a given sensor node to produce their clock estimation. In RBS, a reference node broadcasts reference beacons that serve the nodes in the network to perform receiver-receiver pairwise synchronization. TPSN, however, creates a hierarchical structure in which each node synchronizes to its parent in a sender-receiver fashion. Yet, in PBS, a pair of supernodes $A$ and $P$ exchange messages that are overheard by all nodes in the network, allowing each node to construct their local estimate of the clock offset and skew with respect to the supernodes based on reception time stamps. Thus, for a given node $B$ the quality of offset estimation with respect to reference node $P$ can be obtained as follows [8]:

$$
\operatorname{var}\left(\hat{\theta}_{\text {offset }}^{(B P)}\right) \geq \frac{\sigma^{2} \sum_{i=1}^{\tilde{m}} D_{i}^{2}}{\tilde{m} \sum_{i=1}^{\tilde{m}} D_{i}^{2}-\left(\sum_{i=1}^{\tilde{m}} D_{i}\right)^{2}}
$$

It can be noted from (1) that the estimation quality, represented by the variance of the estimator, depends on the number of received messages $\tilde{m}$ and the time stamps differences $D_{i}$. Increasing $\tilde{m}$ will enhance estimation quality in detriment of the energy consumed; this trade-off will be treated throughout this work in order to provide the basis for its theoretical limits. For simplicity reasons and without loss of generality, the problem of clock offset estimation will be studied in this work, although it can be generalized to skew estimation as well. More recent works such as $[9,10]$ approach the energy efficiency problem from a protocol perspective, without detailing the physical phenomena involved in wireless channels. For example, [9] proposes a new algorithm, the Recursive Time Synchronization Protocol (RTSP), which aims to minimize the number of transmitted messages in a WSN, although the authors do not include in their analysis the transmit or receive power in each sensor node as part of the minimization problem. Thus, many authors expose this energy-synchronization quality balance as a known open topic (such as [11,12]), to the best of our knowledge, previous contributions in the field of clock synchronization focus on the algorithmic aspect of the timing mechanism with little concern on the energy, physical phenomena (i.e., fading channels) and average message delay required to attain such a goal. Moreover, the trade-off "transmit power-clock synchronization quality” is a critical issue for wireless embedded systems that requires to find an optimal solution. This paper extends the work we presented in [13] and provides a concrete answer to the following question: "What is the minimum energy required to synchronize a WSN within a target clock accuracy?"

\section{System Model}

Transmit power and clock synchronization quality operate on different layers: the first one is a physical magnitude whereas the latter belongs to the application layer. Nonetheless, with the introduction of the cross layer design concept [1], all layers should be aligned in order to achieve an energy-optimal solution. However, prior to estimation, physical layer reception occurs with a given probability of failure as a function of the transmit power $S$, given by the outage probability $P_{\text {out }}$ of the channel, defined as the probability that the received signal falls under a minimum acceptable threshold [14]. Some authors prefer to use the term packet reception rate (PRR), which generally refers to the ratio between the received and transmitted packets [15], although in this work it is more convenient to talk of outage probability, since it provides a closer look into the physical effects that lead to successfully receiving a packet or not.

Let's consider each node's clock offset $\theta$ is estimated with an unbiased estimator $\hat{\theta}$, and let $\sigma_{\hat{\theta}}^{2}$ be the variance of the clock offset estimator. The problem described in this section centers on the fact that sender node $A$ sends $m$ packets while receiver node $B$ receives $\tilde{m}=m \cdot\left(1-P_{\text {out }}\right)$ successful messages. The outage probability can also serve as a measure of the delay $\delta$ introduced by the communication channel for achieving the synchronization quality, since the probability

$P_{s}(m, \tilde{m}, p)$ of successfully receiving the target $\tilde{m}$ messages when sending $m>\tilde{m}$ messages is a binomial random variable (RV) with success probability $p=1-P_{\text {out }}$ and mean $E\left[P_{s}(m, \tilde{m}, p)\right]=m \cdot\left(1-P_{\text {out }}\right)$. This leads to an average delay per message $\delta$ defined by the rate of successfully received messages as follows:

$$
\delta=T_{M} \cdot \frac{m}{E\left[P_{s}(m, \tilde{m}, p)\right]}=\frac{T_{M}}{1-P_{\text {out }}}
$$


where $T_{M}$ is the message transmission time. Thus, reducing the outage probability will also enhance the synchronization time. That is, time-sensitive applications may adjust their performance by tuning the transmit power accordingly. However, this must be balanced with the application's energy budget, since in order to reduce $P_{\text {out }}$, the transmit power $S$ must be increased. Considering the estimation quality depends on the number of successfully received packets $\tilde{m}$, the interesting relation $\sigma_{\hat{\theta}}^{2}=f(S)$ is sought. Thus, it is necessary to account for the estimation quality's dependance on the number of received messages, namely $\sigma_{\hat{\theta}}^{2}(\tilde{m})$, and the number of received messages as a function of transmit power, i.e. $\tilde{m}(S)$.

In this work, we analyze two different perturbations of the estimated magnitude, i.e. Gaussian and exponential distributions corresponding to the impairments of the estimated clock offset $\theta$. As explained in [16,17], a single-server $\mathrm{M} / \mathrm{M} / 1$ queue can fittingly represent the $\mathrm{cu}-$ mulative link delay for point-to-point hypothetical reference connection, where the random delays are independently modeled as exponential RVs. The exponential random delays have their origins in the access time and processing times of the nodes. The reason for adopting Gaussian pdf is due to the central limit theorem, which asserts that the pdf of the sum of a large number of independent and identically distributed (iid) RVs approaches that of a Gaussian RV. This model will be appropriate if the delays are thought to be the addition of numerous independent random processes [17]. In addition to this, when nodes are estimating the magnitudes by means of the commonly used technique of Medium Access Control (MAC) layer time-stamping, the estimation noise is Gaussian distributed since the send time, access time and transmission time uncertainties are canceled out $[7,18]$. We also approach the synchronization problem from the local perspective of a node that is synchronizing with a neighbouring node, irrespective of the network size and topology. Still, we consider that the nodes' spatial positions remain approximately constant during the synchronization process, as specified in Section 3.2. The analysis presented in this work is not tied to a particular procedure but it represents a universal lower bound on the “energy_synchronization quality” trade-off.

\subsection{Estimators and Theoretical Limits}

The trade-off studied in this work can be stated as an estimation problem. Both expected value and variance of the offset's unbiased estimator are defined as shown below:

$$
\begin{gathered}
E[\hat{\theta}]=\theta \\
\sigma_{\hat{\theta}}^{2}=E\left[(\hat{\theta}-E[\hat{\theta}])^{2}\right]=E\left[(\hat{\theta}-\theta)^{2}\right]=\sigma_{\hat{\theta}}^{2}(\tilde{m})
\end{gathered}
$$

In order to formulate a general problem, the CramerRao lower bound [19] can be used for delimiting the best performance an estimator can afford. Thus, the estimation quality relates with the Fisher Information function $I[\theta]$ as follows:

$$
\sigma_{\hat{\theta}}^{2} \geq \frac{1}{I(\theta)}
$$

with the Fisher Information's expression as shown below [19]:

$$
I(\theta, \tilde{m}) \triangleq-E\left[\frac{\partial^{2}}{\partial \theta^{2}} \ln f(\theta, \tilde{m})\right]=E\left[\left(\frac{\partial}{\partial \theta} \ln f(\theta, \tilde{m})\right)^{2}\right]
$$

where $f$ is the likelihood function of the parameter $\theta$.

\subsection{Communication Channel Model}

For wireless channels, received to transmitted power ratio $S_{R} / S$ is dictated by [14]:

$$
\frac{S_{R}}{S}(d B)=10 \log K-10 \gamma \log \frac{d}{d_{0}}-\psi_{d B}
$$

where $K$ is a constant that models the antenna gain, $d_{0}$ a reference distance, $\gamma$ the path loss exponent, $d$ the distance between transmitter and receiver nodes, and $\psi_{d B}=10 \log \psi$, being $\psi$ a RV that models either large-scale (shadowing) or small-scale (multipath fading) effects. A communication is defined to be successful, i.e. the receiver can process the transmitted message, when the received Signal-to-Noise Ratio (SNR) $\gamma_{s}$ satisfies $\gamma_{s}>\gamma_{0}$, being $\gamma_{0}$ the minimum acceptable SNR by the receiver [14]. We will consider that the wireless channel is memoryless and time-invariant, meaning that each channel use will be independent and uncorrelated from each other, i.e., they will undergo independent and identically distributed (i.i.d.) fading effects [14]; this means that two subsequent messages sent over the wireless channel will present independent and uncorrelated impairments. Although this situation is not always present in practice, this assumption simplifies the mathematical problem while at the same time enables us to gain insight into the general problem. This condition may be applicable to fast-varying channels as well as moving sensors where the displacement distance $\Delta d$ of a sensor is much less than the distance $d$ that separates a pair of sensors. In a typical scenario where wireless sensors transmit over $2.4 \mathrm{GHz}$, with a wavelength of $\lambda=1.25 \mathrm{~cm}$, it will be sufficient that $\Delta d \approx \lambda$ and $d \geq 1.25 \mathrm{~m}$.

\subsection{Multiple Broadcast Domains}

Figure 1 depicts a situation where nodes deployed in a network contained in different broadcast domains need to 


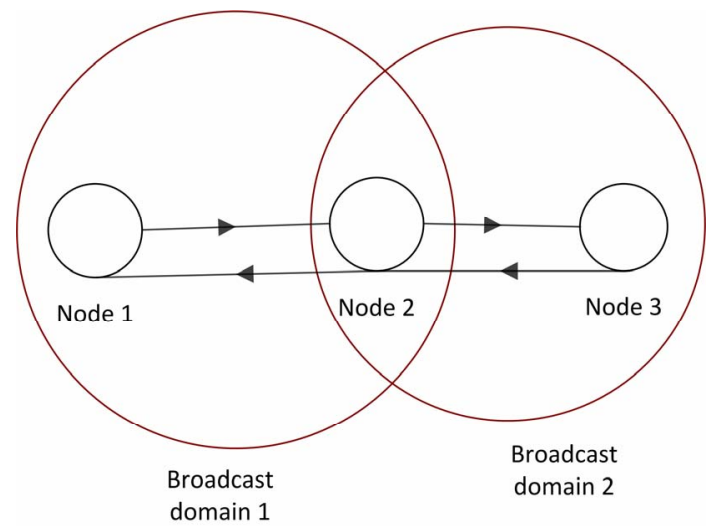

Figure 1. Network topology where multiple broadcast domains are present and multi-hop communication is required.

communicate to each other through an intermediate node. We will initially study the one-way message exchange situation, where Node 1 sends messages to Node 2, and the latter estimates Node 1's clock offset without message exchange. Following to presenting a solution to this problem, we will study the two-way message exchange scenario, where Node 2 will respond messages to Node 1, and they will both estimate each other's clock offsets. Finally, we will extend the results to the case in which Node 1 needs to communicate with Node 3 through Node 2 , which will create a 4-way message exchange (Node 1-Node 2, Node 2-Node 3, and all the return messages to Node 1), a situation that can be generalized to $\mathrm{N}$-way if there are N/2 broadcast domains and all sensors need to communicate with each other in the network. Therefore, with $\mathrm{N}$-way message exchange we address the multiple hop communications in a multi-domain WSN.

\subsection{Problem to Solve: Energy Optimization}

The number of successfully received packets $\tilde{m}$ is related to the transmit power $S$ as shown below:

$$
\tilde{m}=m \cdot\left[1-P_{\text {out }}(S)\right]
$$

The main challenge is to find the transmit power $S$ that satisfies the following condition:

$$
\min (S) \text { s.t. } \sigma_{\hat{\theta}}^{2}(\tilde{m})<\varepsilon
$$

Equation (9) seeks the minimum transmit power $S$ that guarantees the necessary amount of received messages $\tilde{m}$ so that the clock offset estimation error $\sigma_{\hat{\theta}}^{2}$ is less than a desired level $\varepsilon$. For Cramer-Rao efficient estimators, i.e. estimators that attain equality in (5), the following inequality can be stated:

$$
\sigma_{\hat{\theta}}^{2}=\frac{1}{I(\theta, \tilde{m})}<\varepsilon
$$

where $I$ is the Fisher Information of the estimated pa- rameter $\theta$ as a function of the received samples $\tilde{m}$. Thus, the problem can be stated as follows:

$$
\text { Find: } \min (S) \text { s.t. } I(\theta, \tilde{m})>\frac{1}{\varepsilon}
$$

Equation (11) can be thought of as an expression of cross-layer design in wireless sensor networks, since it relates a physical layer magnitude $(S)$ with an application layer parameter $(\varepsilon)$. This expression seeks the minimum transmit power $S$ for achieving a desired estimation error $\varepsilon$ on the clock offset $\theta$ by successfully receiving $\tilde{m}$ messages after transmitting $m$ messages.

In order to account for energy optimization, both transmitter and receiver energy must be minimized; the first one depends on the transmit power $S$ and the number of transmitted messages $m$, whereas the latter is determined by the total time the receiver circuit is powered-on. A priori, the time during which the receiver is turned on could be defined by $\tilde{m} \cdot \delta=m \cdot T_{M}$; however, this time equals the total transmit time, while the receiver node should be turned on for longer in order to receive all the messages since it cannot know in advance each message arrival time. Then, it is expected that the receiver assesses the channel properties and increases its receive time by a linear factor of $1 /\left(1-P_{\text {out }}\right)$. Thus, the total receive time becomes $\tilde{m} \cdot \delta /\left(1-P_{\text {out }}\right)=m \cdot \delta>m \cdot T_{M}$. That said, the total energy function for a pair of nodes $(i$, $j$ ), where node $i$ is transmitting messages to node $j$, can be expressed as follows:

$$
\begin{aligned}
E_{i j}(\text { Total }) & =E_{i}(T x)+E_{j}(R x) \\
& =S \cdot m \cdot T_{M}+\eta \cdot S \cdot m \cdot \delta \\
& =S \cdot m \cdot \delta \cdot\left(\frac{T_{M}}{\delta}+\eta\right) \\
& =S \cdot m \cdot \delta \cdot\left(1+\eta-P_{\text {out }}\right)
\end{aligned}
$$

where $T_{M} / \delta=1-P_{\text {out }}$ as per (2) and $\eta \triangleq S_{R x} / S$ represents the ratio between the receive power and the transmit power, which typically falls in the range $0.5 \sim 0.8$ for commercial transceivers [20]. Although the energy in (12) depends on $\left(1+\eta-P_{\text {out }}\right) \in(\eta, 1+\eta)$, this term has a smooth variation with $S$ for which it does not strongly contribute to the overall variation as the rest of the unknowns $S, m$ and $\delta$ do, which may present an unbounded variation as it will be shown in Section 6 . Hence, it is sufficient to minimize the product of all $S, m$ and $\delta$ to find the minimum energy working point. Then, let:

$$
A(S, m, \delta)=S \cdot m \cdot \delta
$$

be a measure of the energy employed in the synchronization process. Thus, the objective is to minimize the $A(S, m, \delta)$ function for both small-scale and large-scale fading effects. This will be the main motivation 
throughout the rest of this work.

\subsection{Distribution Functions of the Clock Offset}

\subsubsection{Gaussian Distribution Function}

As per (6), the Fisher Information function requires a likelihood function to be applied. Considering the case of Gaussian distributed likelihood functions, for $\tilde{m}$ Gaussian i.i.d observations of $\theta$, the joint probability distribution function is expressed as:

$$
f(\theta, \tilde{m})=\frac{1}{\left(2 \pi \sigma_{V}^{2}\right)^{\tilde{m} / 2}} \exp \left[-\sum_{j=1}^{\tilde{m}} \frac{\left(\theta_{i}-\theta\right)^{2}}{2 \sigma_{V}^{2}}\right]
$$

where $\sigma_{V}^{2}$ is the variance of the perturbations that impair the measurements around the real value of the parameter $\theta$ to be estimated. Operating with (6), (11) and (14), we obtain:

$$
I(\theta, \tilde{m})=\frac{\tilde{m}}{\sigma_{V}^{2}}>\frac{1}{\varepsilon}
$$

\subsubsection{Exponential Distribution Function}

In this section we study the case in which the offset $\theta$ will be estimated from $\tilde{m}$ observations affected by exponential random delays. Let the one-way message estimator of the clock offset be:

$$
\hat{\theta}=\theta+X-\bar{X}
$$

where $X$ is the delay of the channel with exponential pdf, and $\bar{X}$ is the expectation of $X$, thus $\theta$ is an unbiased estimator. The pdf of $X$ can be expressed as:

$$
f_{X}(x)=\lambda \exp (-\lambda x), x \geq 0
$$

where $\bar{X}=1 / \lambda$ and $\sigma_{V}^{2}=1 / \lambda^{2}$ are the expectation and variance of the delay $X$, respectively. The joint pdf after $\tilde{m}$ i.i.d received messages becomes:

$$
f(\theta, \tilde{m})=\lambda^{\tilde{m}} \exp [-\tilde{m} \lambda(\hat{\theta}-\theta-\bar{X})], \hat{\theta}-\theta-\bar{X} \geq 0
$$

By applying (6), the Fisher Information for exponential delays is defined by:

$$
I(\theta, \tilde{m})=\tilde{m}^{2} \lambda^{2}=\frac{\tilde{m}^{2}}{\sigma_{V}^{2}}
$$

For notational simplicity, we will symbolize the case of Gaussian delays with the parameter $\rho=1$ and exponential delays with $\rho=2$.

\section{One-Way Message Clock Offset Estimation Quality as a Function of Transmit Power}

\subsection{Motivation}

Clock estimation by means of unidirectional messages represents an energy-efficient situation for each receiver node, since it does not require to implement a message exchange mechanism, while it can minimize or turn-off its radio operation upon receiving the required number of messages. The Flooding Time Synchronization Protocol (FTSP) [21], makes use of this technique, where each node produces a linear regression estimation of the sender's clock offset. For this work, the one-way message mechanism represents the starting point for exposing the underlying issues associated with clock synchronization by means of wireless messages.

\subsection{Large-Scale Effects: Path Loss and Shadowing}

Large-scale fading represents the average signal power attenuation or path loss over large areas, a phenomenon affected by prominent terrain contours (billboards, clump of buildings, etc.) between the transmitter and receiver [22]; still, for indoor applications, this phenomenon is also present for distances smaller than 10 meters [14]. Under path loss and shadowing, the outage probability $P_{\text {out }}$ is defined as the probability that the received power falls below a given outage threshold $S_{R x}$ expressed in $\mathrm{dBm}$ as found below [14]:

$$
\begin{aligned}
& Q(z) \triangleq \int_{z}^{+\infty} \frac{1}{\sqrt{2 \pi}} \exp \left(-\frac{x^{2}}{2}\right) \mathrm{d} x=\frac{1}{2} \operatorname{erfc}\left(\frac{z}{\sqrt{2}}\right) \\
& z(S)=\frac{S_{R x}-\left[S+10 \log K-10 \gamma \log \left(d / d_{0}\right)\right]}{\sigma_{\psi_{d B}}} \\
& P_{\text {out }}(S)=1-Q(z(S))
\end{aligned}
$$

with the unknown transmit power $S$ expressed in $\mathrm{dBm}$. Parameters $K, \mathrm{~d}, d_{0}, \gamma$, d defined in (7) are assumed known. In this scenario, the RV $\psi_{d B}$ assumes a Gaussian distribution with zero mean and variance $\sigma_{\psi_{d B}}$ (assumed known). Involving (8), (11), (20), (15) for Gaussian delays $(\rho=1)$ and (19) for exponential delays ( $\rho=2)$, it can be seen that for a desired estimation precision $\varepsilon$, the transmit power $S$ must fulfill the following:

$$
\left[m Q\left(\frac{S_{R x}-\left[S+10 \log K-10 \gamma \log \left(d / d_{0}\right)\right]}{\sigma_{\psi_{d B}}}\right)\right]^{\rho}>\frac{\sigma_{V}^{2}}{\varepsilon}
$$

Equation (21) shows that for decreasing estimation error $\varepsilon$, either power $S$ or number of transmitted messages $m$ must be increased accordingly. Being the $Q(z)$ function bounded by the interval $(0,1)$, condition in $(21)$ can be met if and only if $\frac{\sigma_{V}^{2}}{m \cdot \varepsilon} \in(0,1)$. The range of this expression is dominated by $m$ for a given estimation 
error $\varepsilon$. Since $Q$ increases with increasing $S$, the minimum transmit power $S_{\min }$ will be found on the limit of equality in (21). It is then convenient to rewrite this equation into a function as follows:

$$
\left.B\left(S_{\min }, m\right)\right\rfloor_{\varepsilon}=\left[m Q\left(\frac{K_{1}-S_{\min }}{\sigma_{\psi_{d B}}}\right)\right]^{\rho}-\frac{\sigma_{V}^{2}}{\varepsilon}=0
$$

with $K_{1}=S_{R x}-10 \log K+10 \gamma \log \left(d / d_{0}\right)$. From (22), the number of transmitted messages $m$ is determined by:

$$
\left\lceil m_{\min }\right\rceil=\frac{1}{Q\left(\frac{K_{1}-S_{\min }}{\sigma_{\psi_{d B}}}\right)}\left(\frac{\sigma_{V}^{2}}{\varepsilon}\right)^{1 / \rho}
$$

After combining (2) and (20), the delay $\delta$ adopts the following expression under large-scale effects:

$$
\delta_{\min }=\frac{T_{M}}{Q\left(\frac{K_{1}-S_{\min }}{\sigma_{\psi_{d B}}}\right)}
$$

By substituting (23) and (24) into (13), and expressing $S_{\min }$ in $\mathrm{dBm}$, the minimization problem is stated as:

$$
\begin{aligned}
& \frac{\mathrm{d} A}{\mathrm{~d} S_{\min }}=\frac{\mathrm{d}\left(10^{0.1 S_{\min }} \cdot m_{\min } \cdot \delta_{\text {min }}\right)}{\mathrm{d} S_{\min }}=0 \\
& \Rightarrow \frac{\mathrm{d}}{\mathrm{d} S_{\min }}\left[\frac{T_{M} \cdot 10^{0.1 S_{\min }}}{Q^{2}\left(\frac{K_{1}-S_{\min }}{\sigma_{\psi_{d B}}}\right)}\left(\frac{\sigma_{V}^{2}}{\varepsilon}\right)^{1 / \rho}\right]=0
\end{aligned}
$$

Defining $u=\left(K_{1}-S_{\min }\right) / \sigma_{\psi_{d B}}$, we can rewrite (25) as follows:

$$
\frac{2 \cdot Q^{\prime}(u)}{\sigma_{\psi_{d B}} \cdot Q(u)}+0.23=0
$$

Considering $Q^{\prime}(u)=-(2 \pi)^{-1 / 2} \cdot \exp \left(-\frac{u^{2}}{2}\right)$, and replacing back $S_{\min }=K_{1}-\sigma_{\psi_{d B}} \cdot u$, (25) solves to the following condition for both optimized transmit power and transmitted messages:

$$
Q\left(\frac{K_{1}-S_{\min }}{\sigma_{\psi_{d B}}}\right)-\frac{2 \cdot \exp \left[-\frac{1}{2}\left(\frac{K_{1}-S_{\min }}{\sigma_{\psi_{d B}}}\right)^{2}\right]}{0.23 \sqrt{2 \pi} \sigma_{\psi_{d B}}}=0
$$

which can be graphically solved to find the optimal $S_{\min }$ value, provided that $S_{\min } \geq K_{1}$. Equations (23) and (27) represent an energy-efficient solution to the target estimation error $\varepsilon$ under the effect of large-scale fading.

\subsection{Small-Scale Effects: Multipath Fading}

Small scale fading refers to the dramatic changes in signal amplitude and phase as a result of small changes in spatial separation between transmitted and receiver [22], a situation that occurs when the transmitter and receiver nodes are surrounded by a large number of scattering objects [14]. Under small scale fading, or Rayleigh fading, there is no line-of-sight signal component and the envelope of the received signal is described by a Rayleigh probability density function (pdf). The outage probability under Rayleigh fading for a minimum acceptable SNR $\gamma_{0}$ and average transmitted SNR $\bar{\gamma}_{s}$ becomes [14]:

$$
P_{\text {out }}(S)=1-\exp \left(-\frac{\gamma_{0}}{\bar{\gamma}_{s}}\right) \approx 1-\exp \left(-\frac{\gamma_{0} \sigma_{N}^{2}}{S}\right)
$$

where $\gamma_{0}$ and $\bar{\gamma}_{s} \approx S / \sigma_{N}^{2}$ are dimensionless whereas the transmit power $S$ and the noise power $\sigma_{N}^{2}$ are expressed in Watt. Consequently, by combining (15) with (8) and (28), the relation between transmit power $S$, number of transmitted messages $m$ and estimation precision $\varepsilon$ for small-scale effects is described by the following expression:

$$
\left.C\left(S_{\min }, m\right)\right\rfloor_{\varepsilon}=\left[m \cdot \exp \left(-\frac{\gamma_{0} \sigma_{N}^{2}}{S_{\min }}\right)\right]^{\rho}-\frac{\sigma_{V}^{2}}{\varepsilon}=0
$$

As per (29), the number of transmitted messages $m$ is determined by:

$$
m=\left(\frac{\sigma_{V}^{2}}{\varepsilon}\right)^{1 / \rho} \exp \left(\frac{\gamma_{0} \sigma_{N}^{2}}{S_{\min }}\right)
$$

The delay $\delta$ under small-scale effects can be expressed as:

$$
\delta_{\min }=T_{M} \cdot \exp \left(\frac{\gamma_{0} \sigma_{N}^{2}}{S_{\min }}\right)
$$

By substituting (30) and (31) into (13), the minimization problem becomes:

$$
\begin{aligned}
& \frac{\mathrm{d} A}{\mathrm{~d} S_{\text {min }}}=\frac{\mathrm{d}\left(S_{\min } \cdot m_{\min } \cdot \delta_{\text {min }}\right)}{\mathrm{d} S_{\min }}=0 \\
& \Rightarrow \frac{\mathrm{d}}{\mathrm{d} S_{\min }}\left[\left(\frac{\sigma_{V}^{2}}{\varepsilon}\right)^{1 / \rho} T_{M} S_{\min } \exp \left(\frac{2 \gamma_{0} \sigma_{N}^{2}}{S_{\min }}\right)\right]=0
\end{aligned}
$$

where $S_{\min }$ is expressed in Watt. Equation (32) solves to:

$$
S_{\min }=2 \gamma_{0} \sigma_{N}^{2}
$$

Consequently, as per (30), the expression of the minimum number of transmitted messages $m$ results in:

$$
\left\lceil m_{\min }\right\rceil=\mathrm{e}^{1 / 2} \cdot\left(\frac{\sigma_{V}^{2}}{\varepsilon}\right)^{1 / \rho}
$$


Equation (34) shows an inversely proportional dependance on $m$ with estimation quality $\varepsilon$, which exhibits the existing trade-off between estimation quality and number of transmitted messages, and their relation to energy consumption as determined by (13).

\section{Two-Way Message Clock Offset Estimation Quality as a Function of Transmit Power}

\subsection{Motivation}

So far, we have studied the problem of one-way message estimation. In this section, we extend our work presented in [13] to the most commonly used technique for estimating the clock offset (and clock skew as well) in the time synchronization problem in WSN's, i.e., two-way message exchange mechanism; a complete description of this technique can be found in [17] and an application of minimum variance unbiased estimation (MVUE) of the clock offset for the two-way message exchange mechanism is detailed in [23].

\subsection{General Provisions}

We will consider that all nodes are constructively identical, meaning that their minimum acceptable SNR $\gamma_{0}$ will be equal for all of them, and they will transmit signals over a symmetric channel in an environment with equal noise power $\sigma_{N}^{2}$ and $\sigma_{\psi_{d B}}$ for both small-scale and large-scale effects, respectively. In addition to this, we will consider that signals transmitted in uplink or downlink transmission will undergo the same stochastic process for the noise impairments, with equal mean, variance and distribution. In summary:

$$
\sigma_{N_{i}}^{2}=\sigma_{N_{j}}^{2} ; \gamma_{0_{i}}=\gamma_{0_{j}} ; \sigma_{\psi_{d B_{i}}}=\sigma_{\psi_{d B_{j}}} \forall i, j
$$

\subsection{Model Statement}

Let's consider the situation where two nodes $i$ and $j$ produce local estimates of the clock offset based on the number of observations (messages) exchanged amongst them, as it is the case of TPSN or RBS. Although these two synchronization protocols differ in nature, they both exploit two-way messages exchange in order to achieve synchronization. Under a two-way message scenario, each node $i$ will compute a valid sample if and only if 1) it succeeds to deliver a message to node $j$ and, 2) it receives the associated response to this message; this means that both transmitted and received (i.e., replied) messages must be successfully delivered to the destination. To be more specific, let's consider node $i$ sends a message $m_{i j}^{k}$ to node $j$ at time $k$. Node $i$ will wait for the reply message $m_{i j}^{k}$ from node $j$ associated to time slot $k$ for computing its $k$-th estimation sample. This property of the nature of two-way message exchange mechanism that makes two independent messages be chained together to produce a valid estimation sample will be further exploited in the next sections.

\subsection{Problem Outline}

Recalling Section 3.4, we now need to find its counterpart for two-way message exchange. Since each individual node $i$ will process the $k$-th estimation sample based on the message it sent to node $j$ and its associated response, we can state the probability that node $i$ receives a valid sample from its neighbour node $j$ at time $k$ as:

$$
P_{i j \rightarrow j i}^{k}=P\left(s_{i \rightarrow j}^{k} \cap s_{j \rightarrow i}^{k}\right)
$$

where $s_{i \rightarrow j}^{k}$ indicates that node $j$ has successfully received a message sent from node $i$, at time $k$. Since the messages sent by the nodes are independent from the other's messages, and each channel use is also an i.i.d. $\mathrm{RV}$, (36) can be rewritten as:

$$
P_{i j \rightarrow j i}^{k}=P\left(s_{i \rightarrow j}^{k}\right) \cdot P\left(s_{j \rightarrow i}^{k}\right)=\left(1-P_{\text {out }_{i \rightarrow j}}^{k}\right) \cdot\left(1-P_{\text {out }_{j \rightarrow i}}^{k}\right)
$$

Since we assumed identical nodes immersed in a symmetric, time-invariant wireless channel, we can rewrite (37) as follows:

$$
P_{i j \rightarrow j i}^{k} \triangleq \breve{P}_{R T S_{i j}}^{k}=\left[1-P_{\text {out }}(S)\right]^{2} \forall k
$$

where $\breve{P}_{R T S_{i j}}^{k}$ is the round-trip success probability for the round-trip message $k$ between nodes $i$ and $j$ and $P_{\text {out }}(S)$ is the traditional outage probability defined in Section 4 . Equation (38) indicates that, for a given node, the probability of receiving a valid round-trip estimation sample $k$ depends on the square of the outage probability of the wireless channel. Moreover, the large-scale and smallscale effects studied in Sections 4.2 and 4.3 will be have to be reapplied with this new channel success factor. The counterpart of (8) under a two-way message scenario becomes:

$$
\tilde{m}=m \cdot \breve{P}_{R T S}=m \cdot\left[1-P_{\text {out }}(S)\right]^{2}
$$

where $\breve{P}_{R T S}=\breve{P}_{R T S_{i j}}^{k}$ for each $(i, j)$ pair and for all $k$.

\subsection{Large-Scale Effects}

By combining (20) with (38), the round-trip success probability $\breve{P}_{R T S}$ equals $Q(z(S))^{2}$. Hence, this leads the problem under large-scale effects to the following solution:

$$
Q\left(\frac{K_{1}-S_{\min }}{\sigma_{\psi_{d B}}}\right)-\frac{4 \cdot \exp \left[-\frac{1}{2}\left(\frac{K_{1}-S_{\min }}{\sigma_{\psi_{d B}}}\right)^{2}\right]}{0.23 \sqrt{2 \pi} \sigma_{\psi_{d B}}}=0
$$

with $S_{\min } \geq K_{1}$. For the minimum transmit power found 
in (40), the corresponding minimum number of transmitted messages is given by:

$$
\left\lceil m_{\min }\right\rceil=\frac{1}{Q^{2}\left(\frac{K_{1}-S_{\min }}{\sigma_{\psi d B}}\right)}\left(\frac{\sigma_{V}^{2}}{\varepsilon}\right)^{1 / \rho}
$$

Yet, the delay under this scenario is expected to be twice as much the one-way message exchange delay:

$$
\delta_{\min }=\frac{2 T_{M}}{Q^{2}\left(\frac{K_{1}-S_{\min }}{\sigma_{\psi_{d B}}}\right)}
$$

\subsection{Small-Scale Effects}

With the introduction of $\breve{P}_{R T S}$, (32) will have its exponent increased by a factor of 4 , which can be thought of as increasing the noise power $\sigma_{N}^{2}$. Thus, obtaining the tuple ( $S_{\min }, m_{\min }, \delta_{\min }$ ) is straightforward:

$$
\begin{aligned}
& S_{\text {min }}=4 \gamma_{0} \sigma_{N}^{2} \\
& \left\lceil m_{\text {min }}\right\rceil=\mathrm{e}^{1 / 4}\left(\frac{\sigma_{V}^{2}}{\varepsilon}\right)^{1 / \rho} \\
& \delta_{\text {min }}=2 \mathrm{e}^{1 / 4} T_{M}
\end{aligned}
$$

This shows that the effect of small-scale effects for the round-trip message scenario can be linearized to the number of round-trips required for obtaining a single valid message $k$. It is interesting to note that the minimum number of messages $m_{\min }$ remains unchanged with respect to the one-way scenario for equal $\varepsilon$, while the adjusting variable remains the transmit power $S$.

\section{N-way Message Clock Offset Estimation Quality as a Function of Transmit Power}

A generalization of the problem can be easily inferred for both fading scenarios. Let a single estimation sample be composed of $n$ messages exchanged between nodes $i$ and $j$ over a symmetrical stationary channel. A value of $n=1$ represents one-way messages, $n=2$ corresponds to two-way messages, and so on. A situation in which a single node produces a single estimate sample based on $n$ hops will represent a $n$-way message exchange mechanism. Then the following generalization can be stated for each channel fading situation.

\subsection{Large-Scale Effects}

$$
Q\left(\frac{K_{1}-S_{\min }}{\sigma_{\psi_{d B}}}\right)=\frac{2 n \cdot \exp \left[-\frac{1}{2}\left(\frac{K_{1}-S_{\min }}{\sigma_{\psi_{d B}}}\right)^{2}\right]}{0.23 \sqrt{2 \pi} \sigma_{\psi_{d B}}}
$$

$$
\begin{gathered}
\left\lceil m_{\min }\right\rceil=\frac{1}{Q^{n}\left(\frac{K_{1}-S_{\min }}{\sigma_{\psi_{d B}}}\right)}\left(\frac{\sigma_{V}^{2}}{\varepsilon}\right)^{1 / \rho} \\
\delta_{\min }=\frac{T_{M}}{Q^{n}\left(\frac{K_{1}-S_{\min }}{\sigma_{\psi_{d B}}}\right)}
\end{gathered}
$$

\subsection{Small-Scale Effects}

$$
\begin{aligned}
& S_{\text {min }}=2 n \gamma_{0} \sigma_{N}^{2} \\
& \left\lceil m_{\text {min }}\right\rceil=\mathrm{e}^{1 /(2 n)}\left(\frac{\sigma_{V}^{2}}{\varepsilon}\right)^{1 / \rho} \\
& \delta_{\text {min }}=n \mathrm{e}^{1 /(2 n)} T_{M}
\end{aligned}
$$

Equation (44) shows that for increasing $n$, both $m_{\operatorname{mim}}$ and $\delta_{\min }$ grow unbounded since $Q \rightarrow 0^{+}$as $n \rightarrow+\infty$. Thus, $S_{\min }$ needs to be increased in order to keep $Q \rightarrow 1^{-}$, so that $m_{\operatorname{mim}}$ and $\delta_{\min }$ do not experiment an abrupt growth as $n$ is raised. For small-scale fading effects, as shown in (45), $S_{\min }$ increases linearly with $n$ for achieving the target estimation error $\varepsilon$; also, the average message delay $\delta_{\text {min }}$ increases unbounded with $n$, which represents no benefit from the energetic or application standpoint. Finally, the minimum number of messages $m_{\text {mim }}$ mainly depends on and inversely proportional to $\varepsilon$.

\section{Application Example: RBS Algorithm}

In this section, the Reference-Broadcast Synchronization (RBS) [6] protocol will be used as an example to apply the aforementioned theoretical limits. In RBS, a pair of receiver nodes synchronize by estimating each other's relative offsets after receiving a common reference signal which is assumed to arrive simultaneously to all nodes within the same broadcast domain. This approach, called receiver-receiver synchronization, removes the largest nondeterministic part of the receive delay due to the broadcast characteristic of the wireless channel. Thus, each pair of neighbouring nodes $(i, j)$ will exchange their observations of the reference broadcast message and will estimate each other's relative clock offset $\theta_{i j}$ with the following estimator:

$$
\hat{\theta}_{i j}=\frac{1}{\tilde{m}} \sum_{k=1}^{\tilde{m}}\left(T_{j, k}-T_{i, k}\right)
$$

Considering that $T_{j, k}=T_{k}+\theta_{i j}+x_{k}$ and $T_{i, k}=T_{k}+y_{k}$, where $x_{k}$ and $y_{k}$ represent AWGN noise with equal mean and variance, while $T_{k}$ is the absolute time at sample $k$, we can rewrite (46) as follows:

$$
\hat{\theta}_{i j}=\frac{1}{\tilde{m}} \sum_{k=1}^{\tilde{m}}\left(\theta_{i j}+z_{k}\right)
$$


where $z_{k} \triangleq x_{k}-y_{k}$ is a Gaussian RV with distribution $N\left(0, \sigma_{V}^{2}\right)$.

Lemma 1: As per (47), RBS estimates the offset between nodes $i$ and $j$ through the sample mean, which is the Minimum Variance Unbiased Estimator (MVUE) estimator of $\theta_{i j}$, attaining the Cramer-Rao Lower Bound (CRLB) exposed in (10). Then, the general lower bounds shown in Section 3.4 are seamlessly applicable to the RBS protocol.

Proof: an unbiased estimator of the parameter $\theta_{i j}$ based on measurements $x$ that attains the CRLB can be found if and only if [19]:

$$
\frac{\partial \ln p\left(x ; \theta_{i j}\right)}{\partial \theta_{i j}}=I\left(\theta_{i j}\right)\left[g(x)-\theta_{i j}\right]
$$

for some functions $I\left(\theta_{i j}\right)$ and $g(x)$. Still, if $I\left(\theta_{i j}\right)$ and $g(x)$ exist, the CRLB equals $1 / I\left(\theta_{i j}\right)$ and $g\left(\theta_{i j}\right)$ is the MVUE estimator of $\theta_{i j}$. Defining $x \triangleq\left\{x_{k}=\theta_{i j}+z_{k}\right\}$, and involving the likelihood function defined in (14), (48) takes the following expression:

$$
\frac{\partial \ln p\left(x ; \theta_{i j}\right)}{\partial \theta_{i j}}=\frac{\tilde{m}}{\sigma_{V}^{2}}\left[\left(\frac{1}{\tilde{m}} \sum_{k=1}^{\tilde{m}} x_{k}\right)-\theta_{i j}\right]
$$

Equation (49) shows that the CRLB is defined by
$I\left(\theta_{i j}\right)=\tilde{m} / \sigma_{V}^{2}$ while

$g(x)=\frac{1}{\tilde{m}} \sum_{k=1}^{\tilde{m}} x_{k}=\frac{1}{\tilde{m}} \sum_{k=1}^{\tilde{m}}\left(\theta_{i j}+z_{k}\right)$ is the MVUE estimator of $\theta_{i j}$. Thus, by introducing (47), the MVUE estimator of $\theta_{i j}$ is the sample mean. Hence, Lemma 1 is proved.

\section{Simulation Results}

This section exposes the simulations results for typical WSN parameters as referenced in [14] under the most commonly used exchange mechanism, i.e. two-way message exchange $(n=2)$. Figure 2 and 3 depict the dependance of the number of transmitted messages $m$ and the required energy $A(S, m, \delta)$ with transmit power $S$ under the influence of large-scale fading, for Gaussian and exponential delays, respectively, and for different values of the estimation quality $\varepsilon$. The counterpart for smallscale or Rayleigh fading is shown in Figure 4 and 5, for Gaussian and exponential delays, respectively. Results show that it is necessary to finely adjust $S$ to obtain a minimum $m$ so as to minimize the overall synchronization energy.

As the number of hops $n$ increases, the energy minima move towards higher values of $S$ for both fading scenar-

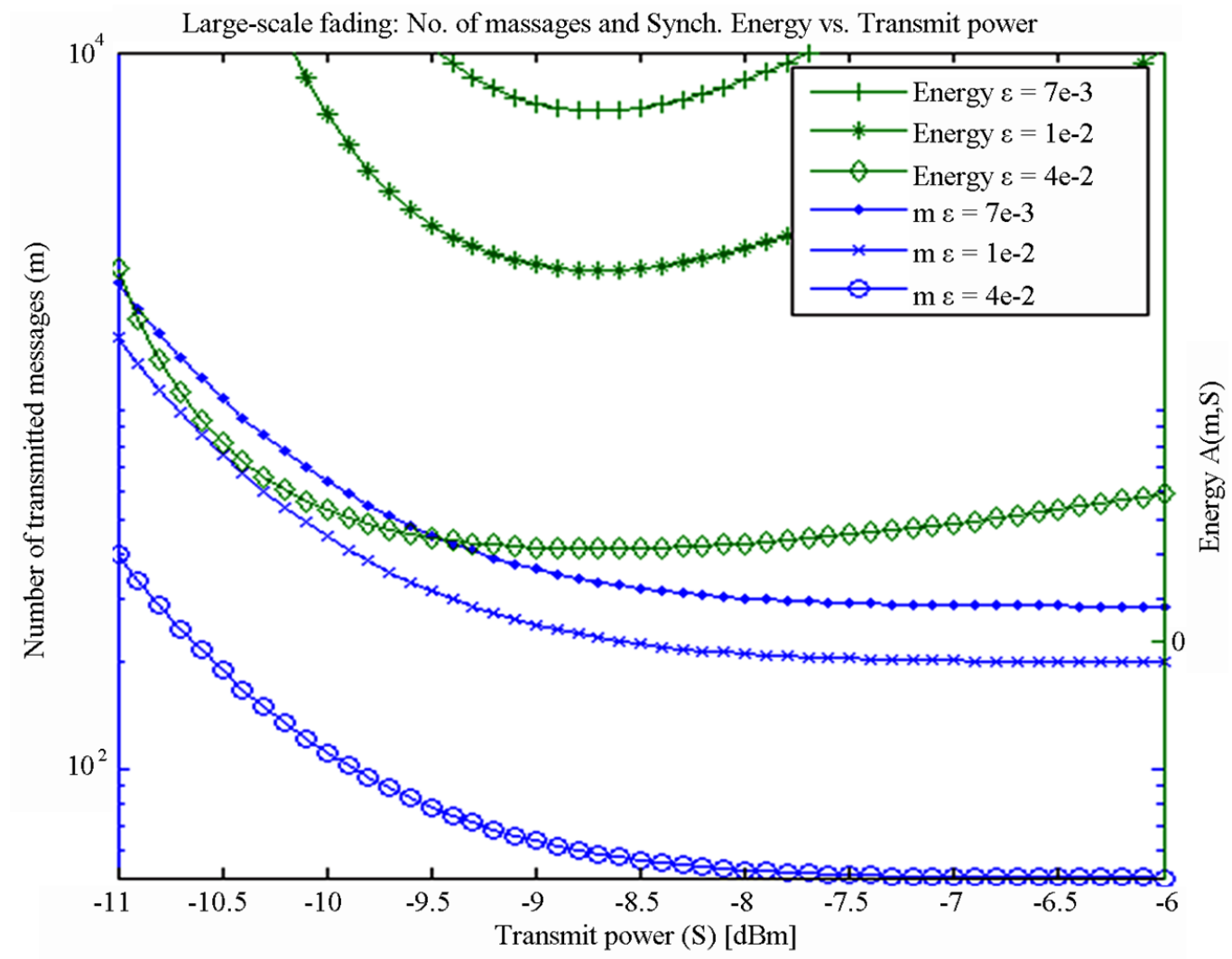

Figure 2. Number of transmitted messages and Energy required as a function of transmit power for large-scale fading, for different clock offset estimation qualities using two-way messages, with Gaussian delays. 


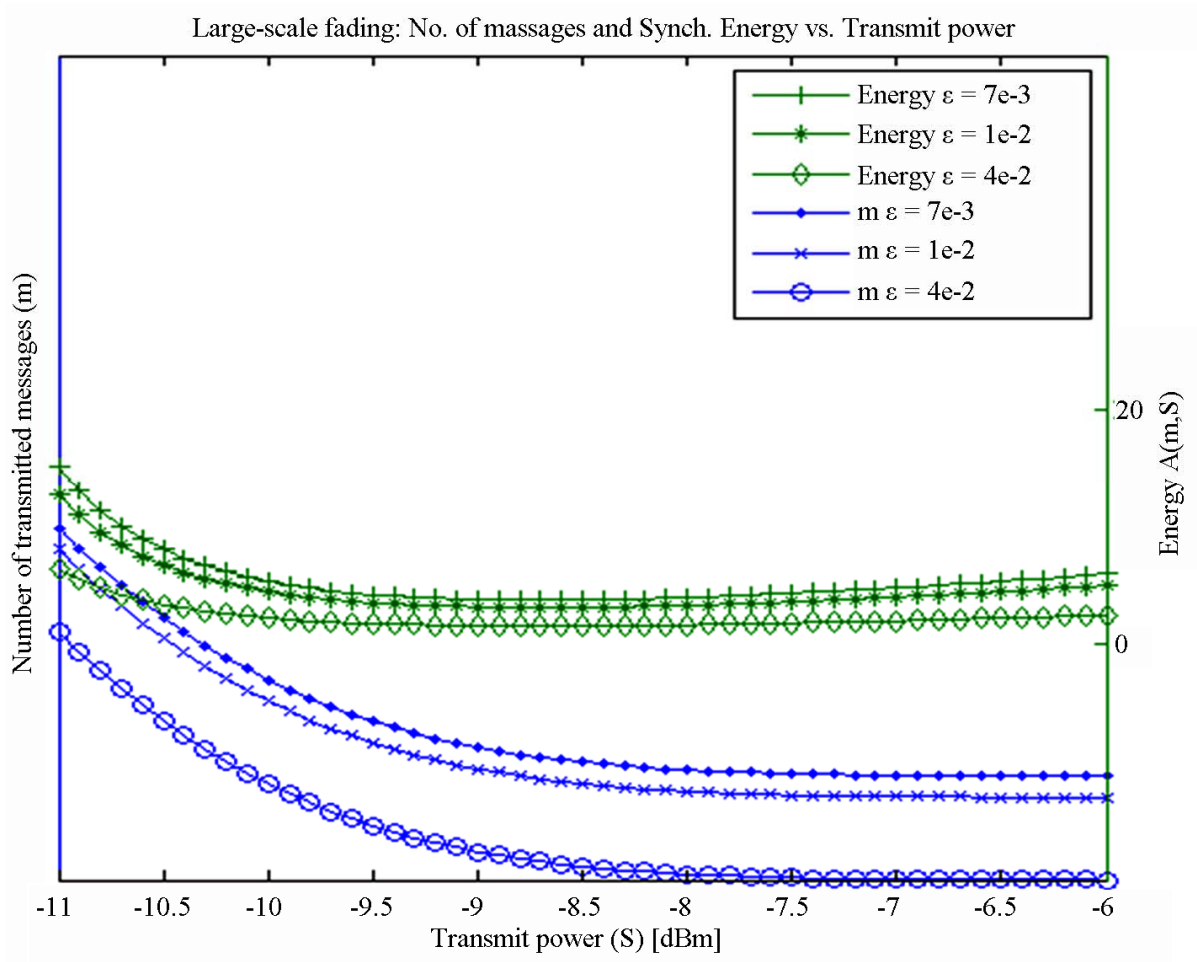

Figure 3. Number of transmitted messages and energy required as a function of transmit power for large-scale fading, for different clock offset estimation qualities using two-way messages, with exponential delays.

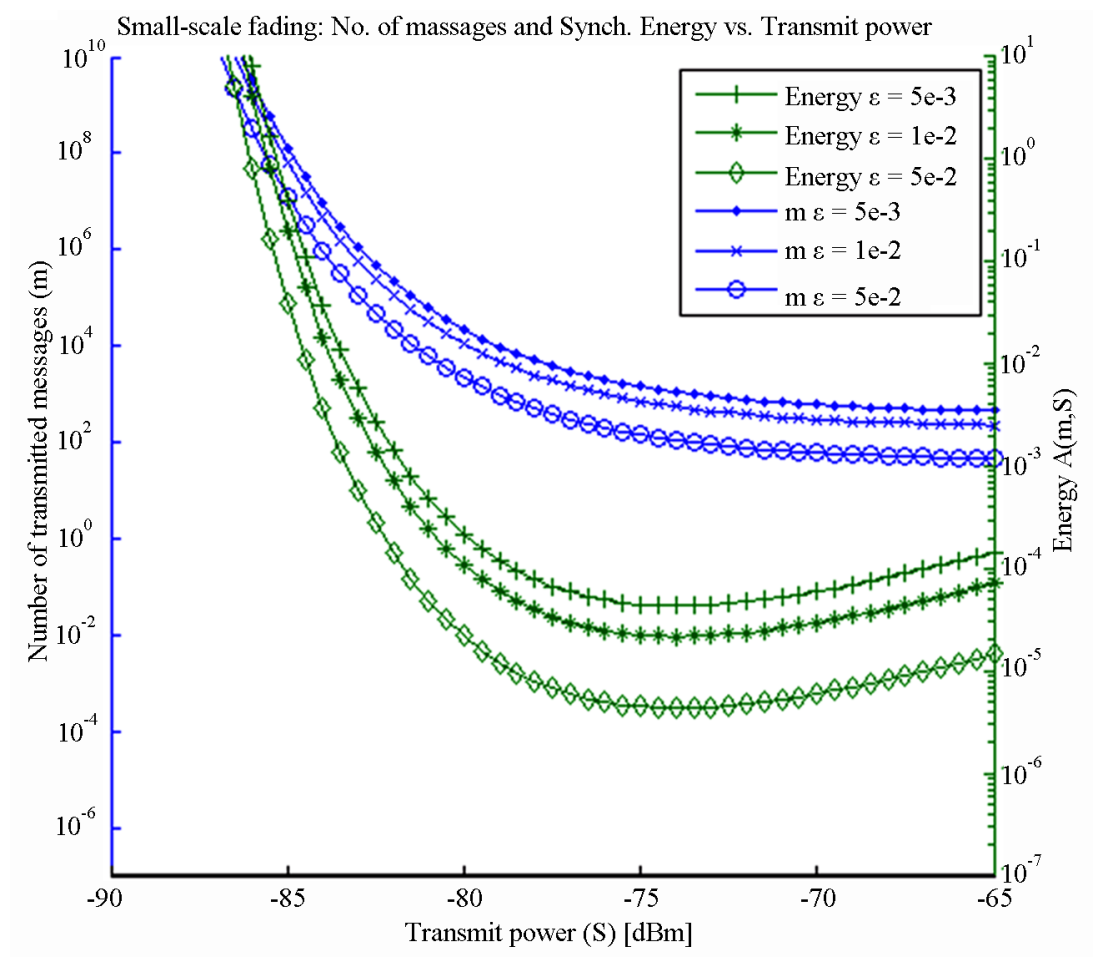

Figure 4. Number of transmitted messages and energy required as a function of transmit power for small-scale fading, for different clock offset estimation qualities using two-way messages, with Gaussian delays.

ios; this means that dense multi-hop sensor networks (large $n$ ) will require more synchronization energy. Ex- amples of these types of networks can be found in [24], applied to farming and geographic surveillance. Figure 6 


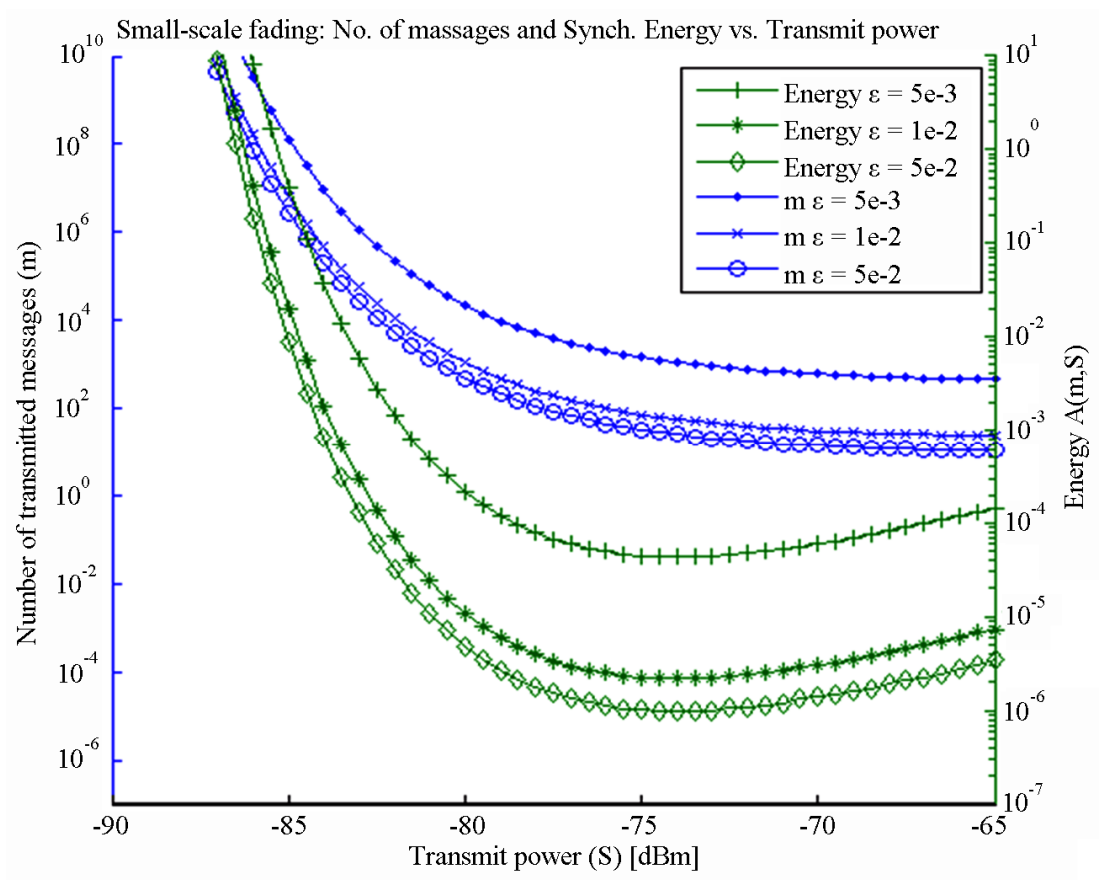

Figure 5. Number of transmitted messages and energy required as a function of transmit power for small-scale fading, for different clock offset estimation qualities using two-way messages, with exponential delays.

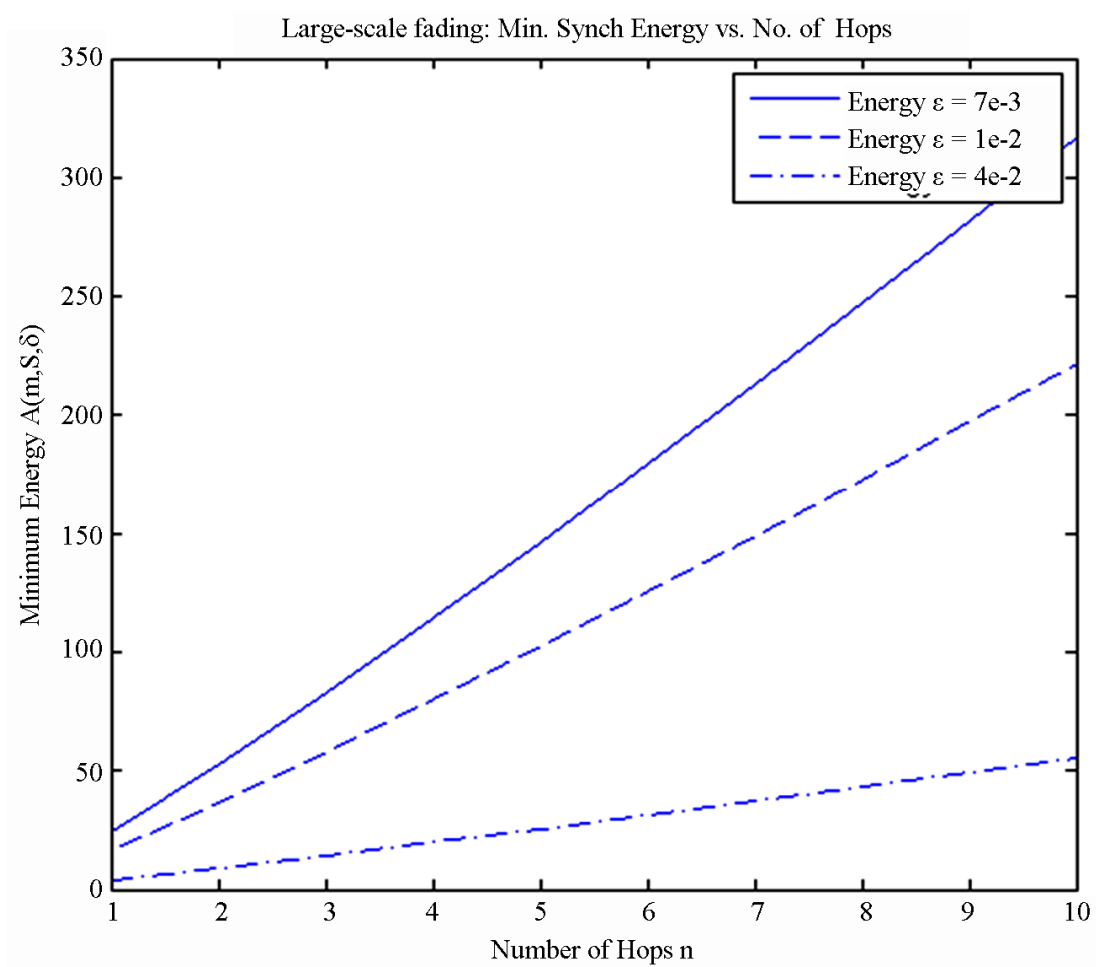

Figure 6. Minimum synchronization energy as a function of number of hops for large-scale fading, for different clock offset estimation qualities, with Gaussian delays.

shows the energy minima for large-scale fading and Gaussian noise, where it can be seen that the growth of $A(S, m, \delta)$ is fairly smooth due to the fact that $S_{\min }$ tends to maximize the Q-function for maintaining $m_{\min }$ and $\delta_{\min }$ as minimum as possible. The counterpart for small-scale fading and Gaussian noise is displayed in Figure 7, where the best-effort situation exhibits a linear growth of the energy function with the number of hops; 


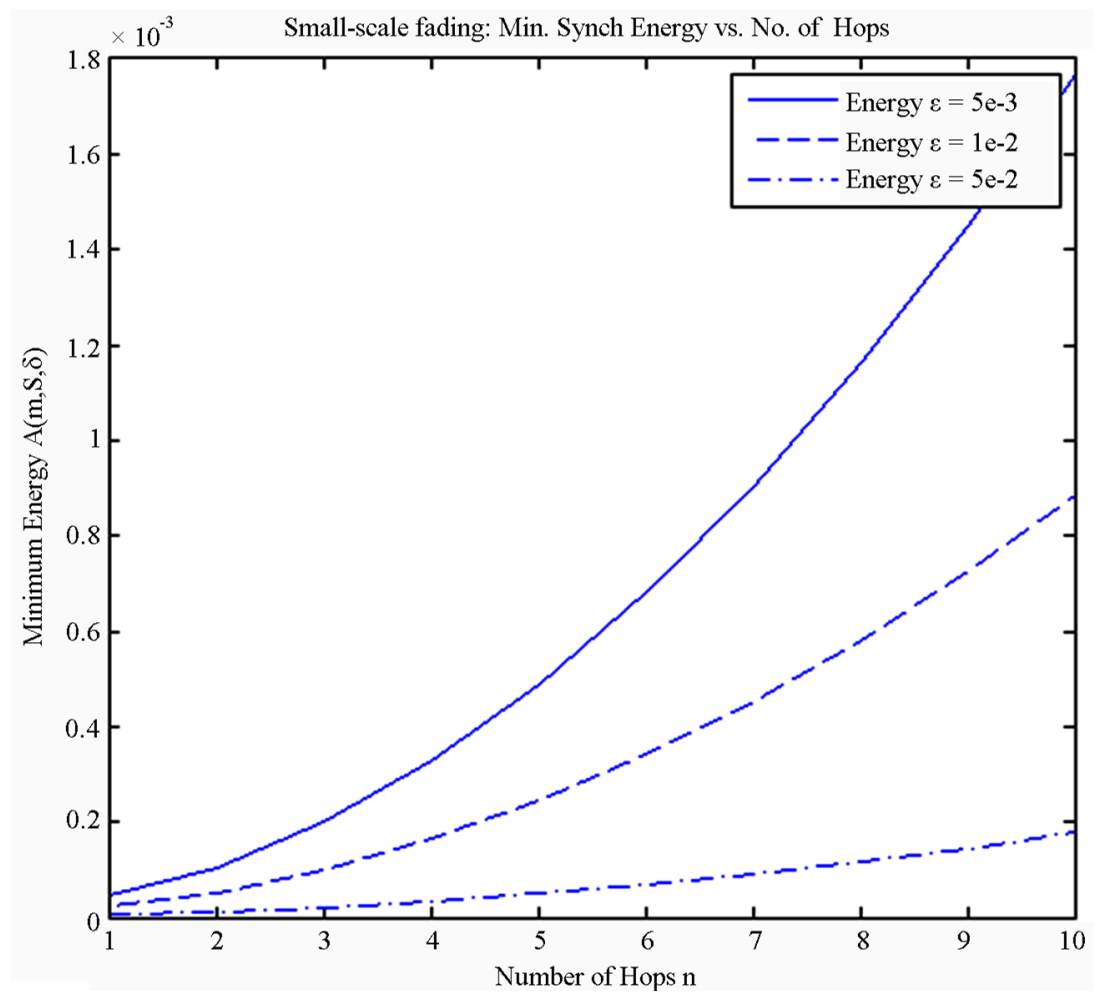

Figure 7. Minimum synchronization energy as a function of number of hops for small-scale fading, for different clock offset estimation qualities, with Gaussian delays.

for values of the transmit power smaller than the minimum-energy power $S_{\min }$, an exponential growth of $A(S, m, \delta)$ is expected for increasing $n$, as dictated by (45).

\section{Summary and Discussions}

Time synchronization for a WSN can be achieved by means of parameter estimation techniques which require a number of messages to be transmitted from a sender to a receiver node. The minimum amount of total energy required for achieving a desired estimation quality $\varepsilon$ is represented by the product of the transmit power $S$, number of messages $m$ and the average message delay $\delta$. By introducing the concept of outage probability of the wireless channel for both large-scale and small-scale fading scenarios, a minimization problem can be stated for the total energy function. The resolution of the entire system finds the energy-optimal working point which represents a lower bound for the estimation quality.

The general results obtained in this work have been applied to the cases of Gaussian and exponential perturbations for the Cramer-Rao efficient, unbiased offset estimator $\hat{\theta}$. For other estimators that do not fulfill these conditions, the estimation error $\sigma_{\hat{\theta}}^{2}$ shall be used instead of the Fisher Information function in order to compute the theoretical limits for that particular case. The renowned one-way and two-way message exchange scenarios have been extensively analyzed in this work, proving that the RBS synchronization algorithm achieves one-way message exchange theoretical limits. Finally, the results obtained throughout this work have been extended for the case of n-way messages, which will serve as a basis to generalize the synchronization energy problem to densely deployed multi-hop wireless sensor networks, as it will be in the realm of the Internet of Things. As part of our future work, we will extend this analysis to a generic WSN aiming to find the network-wide energy vs. estimation quality trade-off, including the effect of interference from simultaneous transmitters in the analysis. We are also considering incorporating in our analysis the development of energy harvesting techniques in an attempt to render the synchronization process as autonomous as possible from energy provided from external environment.

\section{REFERENCES}

[1] A. Swami, Q. Zhao, Y. Hong and L. Tong, "Wireless Sensor Networks, Signal Processing and Communications Perspectives,” Wiley, Hoboken, 2007, pp. 9-89.

[2] T. Locher, P. Von Rickenbach and R. Wattenhofer, "Sensor Networks Continue to Puzzle: Selected Open Problems," Proceedings of the Ninth International Conference on Distributed Computing and Networking, 2008. http://dx.doi.org/10.1007/978-3-540-77444-0_3 
[3] Postscapes, “Tracking the Internet of Things,” 2012. www.postscapes.com

[4] F. Ren, C. Lin and F. Liu, "Self-Correcting Time Synchronization Using Reference Broadcast in Wireless Sensor Network,” IEEE Wireless Communications, 2008.

[5] L. Schenato and G. Gamba, “A Distributed Consensus Protocol for Clock Synchronization in Wireless Sensor Network," Proceedings of the 46th IEEE Conference on Decision and Control, New Orleans, 12-14 December 2007, pp. 2289-2294.

[6] J. Elson, L. Girod and D. Estrin, "Fine-Grained Network Time Synchronization Using Reference Broadcasts," Proceedings of the 5th Symposium on Operating Systems Design and Implementation, Boston, Vol. 36, No. SI, 2002, pp. 147-163.

[7] S. Ganeriwal, R. Kumar and M. Srivastava, "TimingSync Protocol for Sensor Networks,” SenSys '03, ProCeedings of the 1st International Conference on Embedded Networked Sensor Systems, Los Angeles, 2003, pp. 138-149.

[8] K. Noh, E. Serpedin and K. Qaraqe, "A New Approach for Time Synchronization in Wireless Sensor Networks: Pairwise Broadcast Synchronization,” IEEE Transactions on Wireless Communications, Vol. 7, No. 9, 2008.

[9] M. Akhlaq and T. R. Sheltami, "Rtsp: An Accurate and Energy-Efficient Protocol for Clock Synchronization in WSNs," IEEE Transactions on Instrumentation and Measurement, Vol. 62, No. 3, 2013, pp. 578-589.

[10] F. Ferrari, M. Zimmerling, L. Thiele and O. Saukh, "Efficient Network Flooding and Time Synchronization with Glossy," IEEE 10th International Conference on Information Processing in Sensor Networks (IPSN), Chicago, 12-14 April 2011, pp. 73-84.

[11] E. Serpedin and Q. Chaudhari, "Synchronization in Wireless Sensor Networks: Parameter Estimation, Performance Benchmarks, and Protocols,” Cambridge University Press, Cambridge, 2009. http://dx.doi.org/10.1017/CBO9780511627194

[12] Q. Chaudhari, E. Serpedin and K. Qaraqe, “Cramer-Rao Lower Bound for the Clock Offset of Silent Nodes Synchronizing through a General Sender Receiver Protocol in Wireless Sensor Nets,” 16th European Signal Processing Conference (EUSIPCO), Lausanne, 2008.

[13] P. Briff, F. Vargas, A. Lutenberg and L. Rey Vega, “On the Trade-Off of Power Consumption and Time Synchronization Quality in Wireless Sensor Networks," Proceedings of the 11th IEEE Conference on Sensors, Taipei,
28-31 October 2012, pp. 1927-1930.

[14] A. Goldsmith, “Wireless Communications,” Cambridge University Press, Cambridge, 2005. http://dx.doi.org/10.1017/CBO9780511841224

[15] N. Pustchi and T. Korkmaz, "Improving Packet Reception Rate for Mobile Sinks in Wireless Sensor Networks," 2012 IEEE International Symposium on World of Wireless, Mobile and Multimedia Networks (WoWMoM), San Francisco, 25-28 June 2012, pp. 1-9.

[16] D. R. Jeske, "On Maximum-Likelihood Estimation of Clock Offset," IEEE Transactions on Communications, Vol. 53, No. 1, 2005, pp. 53-54. http://dx.doi.org/10.1109/TCOMM.2004.840668

[17] K. Noh, Q. Chaudhari, E. Serpedin and B. Sutter, "Novel Clock Phase Offset and Skew Estimation Using TwoWay Timing Message Exchanges for Wireless Sensor Networks,” IEEE Transactions on Communications, Vol. 55, No. 4, 2007, pp. 766-777. http://dx.doi.org/10.1109/TCOMM.2007.894102

[18] S. Song, L. He, Y. Jiang, C. Hu and Y. Cao, "Wireless Sensor Network Time Synchronization Algorithm Based on sfd," Advances in Wireless Sensor Networks, 2013, pp. 393-400.

[19] S. Kay, "Fundamentals of Statistical Signal Processing: Volume 1,” Prentice Hall, Upper Saddle River, 1993, pp. 27-77.

[20] Texas Instruments Inc., "Cc2520 Data Sheet 2.4 ghz IEEE 802.15.4/Zigbee rf Transceiver,” Texas Instruments Inc., 2007.

[21] M. Maroti, B. Kusy, G. Simon and A. Ledeczi, "The Flooding Time Synchronization Protocol," Proceedings of 2 nd International Conference on Embedded Networked Sensor Systems, 2004, pp. 39-49. http://dx.doi.org/10.1145/1031495.1031501

[22] B. Sklar, "Rayleigh Fading Channels in Mobile Digital Communication Systems-Part 1: Characterization,” IEEE Communications Magazine, Vol. 35, No. 9, 1997, pp. 136-146. http://dx.doi.org/10.1109/35.620535

[23] Q. Chaudhari, E. Serpedin and K. Qaraqe, “On Minimum Variance Unbiased Estimation of Clock Offset in a TwoWay Message Exchange Mechanism,” IEEE Transactions on Information Theory, Vol. 56, No. 6, 2010, pp. 28932904. http://dx.doi.org/10.1109/TIT.2010.2046233

[24] K. Romer and F. Mattern, “The Design Space of Wireless Sensor Networks,” IEEE Wireless Communication, Vol. 11, No. 6, 2004, pp. 54-61. 